Rationale for a Spallation Neutron Source

Target System Test Facility at the

1-MW Long-Pulse Spallation Source

Walter F. Sommer 


\section{DISCLAMMER}

Portions of this document may be illegible in electronic image products. Images are produced from the best available original document. 



\section{Table of Contents}

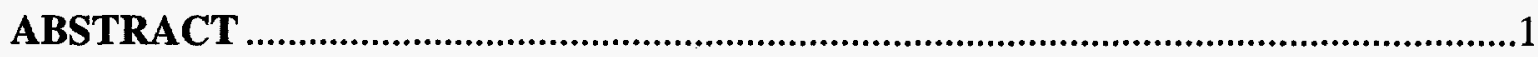

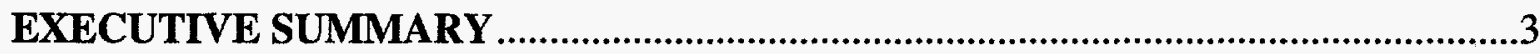

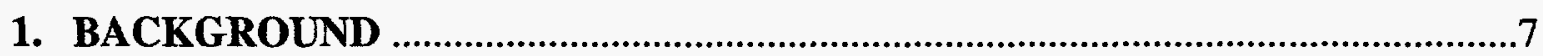

2. SYSTEMS PERFORMANCE ISSUES ........................................................

2.A. Thermal Hydraulics .........................................................................

2.B. Corrosion in Aqueous Systems-Materials Compatibility ...............................8

2.C. Corrosion in Liquid Metal Systems-Materials Compatibility ...........................9

2.D. Corrosion in Molten Salt Systems-Materials Compatibility .............................9

2.E. Contamination of Fluid Systems with Transmutation Products ...........................9

2.F. Development of Surveillance Methods and Monitors of System Performance

2.G. In situ Determination of Heat Transfer Properties and Degradation with Time.

2.H. Comparison of System Design with Performance-Refinement of Design Technique

2.I. Development of Choppers and Other Equipment at Near-Target Locations ......10

2.J. Development of Beam Diagnostics Equipment.

3. MATERIALS PERFORMANCE ISSUES .11

3.A. Mechanical Properties Degradation .11

3.B. Physical Properties Degradation. .11

3.C. Dimensional Stability .11

3.D. Thermal Stress from Thermal Strain at Low Frequency ..................................12

3.E. Shock-Related Stress from Short-Pulse, High-Intensity Operation ...................12

3.F. Synergetic Effects of Radiation and Environment .........................................12

3.G. Comparison of Spallation Neutron Source Performance Data to Fission Reactor Data

3.H. Basic Understanding of Atom Displacement Physics at High Recoil Energies

3.I. Basic Understanding of Transmutation Product Morphology and the Effect on Properties

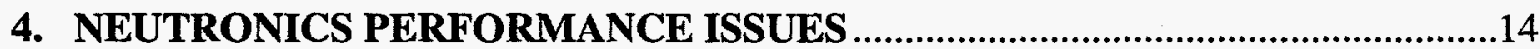

4.A. Production of Neutrons/Proton for Several Materials ......................................14

4.B. Production of Tritons/Proton for Several Materials .........................................14 
4.C. Moderator Design Developments/Ultracold Neutron Source .............................14

4.D. Poison and Decoupling Studies .....................................................................14

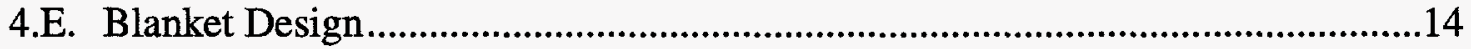

4.F. Transmutation Product Generation Measurements at High Fluence with Burnup and General Neutronics Code Verification and Benchmark Studies

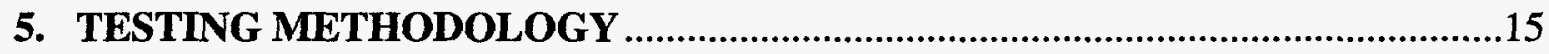

5.A. Short-term Performance Tests ..................................................................15

5.B. Long-term Performance Tests .............................................................18

5.C. Very Long-term Materials Exposure for Radiation Damage Effects Determination

5.D. Use of Instruments at LPSS and MLNSC for Materials Characterization after Irradiation

6. SPALLATION NEUTRON SOURCES UNDER STUDY AND DEVELOPMENT

6.A. Research Facilities

6.A.1. European Spallation Source (ESS)

6.A.2. Short-Pulse Spallation Source (SPSS)

6.A.3. Long-Pulse Spallation Source (LPSS)

6.A.4. Target System Development at the Manuel Lujan Jr. Neutron Scattering Center (MLNSC)

6.A.5. Target System Development at the Swiss Spallation Neutron Source (SINQ)

6.A.6. Target System Development at ISIS at Rutherford-Appleton Laboratory

6.A.7. Intense Proton-Neutron Source (IPNS) Upgrade

6.A.8. Conceptual Design for the Austrian Spallation Source (AUSTRON).....21

6.A.9. Engineering Test Accelerator (ETA)

6.B. Large-Scale Production Facilities .22

6.B.1. Accelerator Production of Tritium (APT) .22

6.B.2. Accelerator-Driven Transmutation Technologies (ADTT)

6.C. Workshop on Materials for Spallation Neutron Sources, Los Alamos, February 1995

7. REFERENCES

8. ACRONYM LIST 


\title{
Rationale for a Spallation Neutron Source Target System Test Facility at the 1-MW Long-Pulse Spallation Source
}

by

Walter F. Sommer

\begin{abstract}
The conceptual design study for a 1-MW Long-Pulse Spallation Source at the Los Alamos Neutron Science Center has shown the feasibility of including a spallation neutron test facility at a relatively low cost. This document presents a rationale for developing such a test bed. Currently, neutron scattering facilities operate at a maximum power of $0.2 \mathrm{MW}$. Proposed new designs call for power levels as high as $10 \mathrm{MW}$, and future transmutation activities may require as much as $200 \mathrm{MW}$. A test bed will allow assessment of target neutronics; thermal hydraulics; remote handling; mechanical structure; corrosion in aqueous, non-aqueous, liquid metal, and molten salt systems; thermal shock on systems and system components; and materials for target systems. Reliable data in these areas are crucial to the safe and reliable operation of new highpower facilities. These tests will provide data useful not only to spallation neutron sources proposed or under development, but also to other projects in accelerator-driven transmutation technologies such as the production of tritium.
\end{abstract}




\section{EXECUTIVE SUMMARY}

This document presents a rationale for developing a test bed for advanced spallation neutron sources. Developmental studies on the neutronics, thermal hydraulics, remote handling, mechanical structure, corrosion in aqueous and non-aqueous systems, and materials for target systems are crucial to ensure safe and reliable operation of new highpower facilities. A test bed can offer wide flexibility in test assemblies and in the variety of studies that are possible. The incremental cost associated with including this facility with the proposed Long-Pulse Spallation Source (LPSS), which uses the Los Alamos Neutron Science Center (LANSCE) accelerator, is very modest. The opportunity to test target assemblies and associated support systems and to perform studies on materials performance to high fluence is crucial because failures at an operating facility are generally extremely costly in terms of time as well as funds.

The maximum power at spallation neutron sources presently in operation at neutron scattering facilities is less than 0.2 MW. New designs are being proposed that call for power levels of 1 to $5 \mathrm{MW}$ and as high as $10 \mathrm{MW}$. Other applications for spallation neutron source systems include facilities for the transmutation of radioactive waste and production of tritium; the designs of these facilities are for power levels approaching 200 MW. Facility designs must take into consideration past experience at spallation neutron source facilities in terms of average specific power density, as well as instantaneous power density when the beam is delivered in very short pulses. As the total power in these facilities increases, the mechanical and control equipment that provides target system coolant and control becomes complex and more expensive, and the limits on materials in terms of radiation damage resistance are approached.

Review committees studying proposals for advanced spallation neutron sources for research and proposals for transmutation technologies have stressed the need for facilities capable of operating for long periods each year at high reliability and availability. Long, reliable operating periods are important, not only to establish an attractive return on the investment of building the facility, but also because a very large number of 1- to 2-day experiments need to be performed at the facility and schedules must be set and adhered to. Because some targets used for neutron scattering experiments have a finite lifetime and are expensive to produce, reliability becomes increasingly important. Reliability is also important as a facility becomes national and international in scope, and transport of equipment and personnel over long distances becomes necessary. The efficiency of the 
facilities currently being studied for transmutation technologies is proportional to the availability and operation time of the entire complex.

There have been numerous operational difficulties at spallation neutron source facilities, particularly in the performance of targets and moderators and the systems that support these units. Many failures have occurred after short operating periods of a month or a few months. From the standpoint of radiation damage effects, target failures have occurred at modest fluences, in some cases less than $10^{21}$ protons $/ \mathrm{cm}^{2}[1,2]$. As a point of comparison, new designs for spallation neutron sources will subject targets to a fluence approaching $10^{22}$ protons $/ \mathrm{cm}^{2}$ in a full-power year [3]. In addition, structural materials around the target system are exposed to a high neutron flux; the fluence on these components will approach $10^{22}$ neutrons $/ \mathrm{cm}^{2}$ in a full-power year [3]. Several studies on the performance of materials in spallation neutron sources have been completed, though very little post-irradiation examination of components used in service at such facilities has been performed. Specially designed experiments aimed at measurement of basic mechanical and physical property changes due to radiation have been limited to low dose, generally less than $10^{21}$ particles $/ \mathrm{cm}^{2}$. The results of these studies are largely phenomenological. Materials radiation effects data from fission reactor studies cannot be directly applied to spallation neutron source applications because the higher energy particles at spallation neutron sources produce higher energy recoil atoms and atom displacement cascades and 10 to 100 times more impurities than in a fission reactor environment. These impurities, especially $\mathrm{He}$ and in some cases $\mathrm{H}$, have a drastic effect on the degradation of materials mechanical properties. A database for high-fluence materials radiation damage performance in spallation neutron source environments is essential for use in future designs that demand reliable operation at high availability.

Control of contamination resulting from radioactive materials is a requirement at all spallation neutron source facilities. In addition to overall target system performance characterization studies, a test bed facility will also support the development of filtration systems, methods for monitoring the chemistry of the coolant systems, and techniques for mitigating the corrosion that results from the interaction of the charged particle beam with the coolant and materials.

Complete target systems can be placed in a test bed for studying neutronic performance at high flux. Well-designed experiments can result in the systematic refinement of the 
detailed design of targets and moderators and their spatial relationship, thus leading to optimized systems.

Code validation at high flux and fluence can be pursued at a test bed facility. Areas to examine include the study of transmutation product buildup and atom depletion and their effect on system radioisotope inventory and neutronic performance. Such studies would compare calculated and measured results.

A test bed facility is useful not only for the development of spallation neutron source target systems for neutron scattering research, but also for the development of target and blanket concepts for the Accelerator Production of Tritium (APT) project, where ${ }^{3} \mathrm{He}$ is to be transmuted to tritium. A similar application is the Accelerator-Driven Transmutation Technologies (ADTT) project, where radioactive waste is to be transmuted to shorter-lived isotopes and $\mathrm{Pu}$ is to be used as the fuel for a subcritical, accelerator-driven, energy-producing assembly.

In summary, neutronic performance studies, as well as systems performance and materials lifetime studies, can be accomplished at a spallation neutron source target system test bed. These studies can lead to optimized target system designs and target system components before the final facility design is completed. Designers, users, and sponsors of the proposed facility can then plan extensive research programs with confidence. 


\section{BACKGROUND}

Advanced spallation neutron sources must operate at increasingly higher power with higher availability for longer periods of time. In general, power will increase from 100 to $200 \mathrm{~kW}$ to 1,5 , or $10 \mathrm{MW}$. The desired availability is in the $80 \%$ to $90 \%$ range. The period of operation for such facilities will increase from 3 to 6 months per year to 9 months per year. These advanced performance requirements point to a need for testing new target systems before they are placed in a production facility.

The conceptual design study for a 1-MW Long-Pulse Spallation Source (LPSS) using the Los Alamos Neutron Science Center (LANSCE) accelerator has shown the feasibility of including a spallation neutron source development test bed at relatively low cost. This test bed will be large in volume and able to accommodate complete target system components. It will allow for integral tests of the target neutronics performance, thermal hydraulics performance, and materials compatibility and performance in a radiation environment.

\section{SYSTEMS PERFORMANCE ISSUES}

All systems that operate in the target cells at spallation neutron sources are subjected to the synergistic effects of energetic particle radiation, cyclic thermal strain and stress, and a variety of chemical states in aqueous systems. Testing components without radiation can give some indication of system performance; however, experience has shown that the synergistic effects added by radiation can produce significant differences in system performance. Specific areas of concern and a description of needed testing are outlined below.

\section{A. Thermal Hydraulics}

The cooling characteristics of high-power spallation neutron source targets are often limited by the magnitude of the surface heat flux from a component. In water-cooled systems, designs are optimized to a coolant flow that ensures that a suitable margin exists between a metal surface temperature and the temperature that leads to coolant boiling (which generally is to be avoided). As the coolant chemistry changes, the metal surfaces may become coated with corrosion products that decrease heat transfer and increase surface temperature. Instrumented in situ tests in a prototypic environment at a test bed 
facility can be performed to determine the magnitude of this corrosive effect and to characterize means of mitigating the effect through coolant chemistry control. Operating experience with pumps, filters, instrumentation, and controls can also be obtained.

Liquid metal systems of $\mathrm{Pb}, \mathrm{Hg}$, or lead-bismuth are considered viable candidates for spallation neutron sources and are often considered necessary for system powers of $2 \mathrm{MW}$ and above for configurations designed to provide neutrons for scattering experiments. The test bed can be configured to permit testing of the entire target system, including flow patterns, pump performance, heat exchanger performance, and metal transport system performance. Measurements can be made with a concurrent prototypic proton beam environment. On-line studies can be made to determine the production of volatile transmutation products and techniques can be developed for removing them. This is important to the requirement for establishing the overall safety of a liquid metal target system. Methods of removing mobile solid transmutation products can also be developed. Such methods would enable the control, for example, of the concentration of Po produced from neutron capture on $\mathrm{Bi}$ (an $\alpha$ particle emitter during radioactive decay) to an acceptable level. The test bed can be a crucial component of the development and acceptance program for liquid metal targets.

Neutron-moderating blankets and reaction zones of $\mathrm{BeF}, \mathrm{LiF}$, and $\mathrm{Pu}$ molten salts are being considered in accelerator-based transmutation technologies that propose to produce energy using a subcritical blanket. Again, the test bed offers the opportunity to study the containment of the salts, the effect of energetic neutrons from a spallation target on the chemistry of the salts, the radionuclide production, and the energy production level in the system, all in a prototypic radiation environment. When coupled to a prototypic target, the operating parameters for these systems, including energy production level and efficiency, can be determined from scaled tests at $1 \mathrm{MW}$; the actual systems may operate at power levels of $200 \mathrm{MW}$ and higher.

\section{B. Corrosion in Aqueous Systems-Materials Compatibility}

Corrosion of materials and re-deposition of corrosion products have caused difficulties at operating spallation neutron source facilities [4]. The physics and chemistry governing corrosion in an environment that includes energetic charged particles has been postulated, but definitive in situ tests have not been performed [5]. A test bed is ideally suited for this purpose. Instrumented coolant loops can be tested with a variety of candidate target and structural materials. Corrosion potentials and rates can be determined. Alterations to the 
coolant chemistry that mitigate the corrosion can also be evaluated. Eliminating the concern about corrosion effects in spallation neutron source systems would be a significant contribution to development of advanced facilities operating at high availability. Any proposed new system could be tested at the test bed.

\section{C. Corrosion in Liquid Metal Systems-Materials Compatibility}

In conjunction with the loop tests for liquid metal target systems described above, surveillance of the impurity content of the liquid during irradiation, and post-irradiation examination of the materials used as containers, will quantify corrosion rates and lead to designs that exhibit long potential service times. New concepts can be conveniently studied in the test bed before they are placed in service.

\section{D. Corrosion in Molten Salt Systems-Materials Compatibility}

In a manner similar to that proposed for liquid metal targets and in conjunction with the molten salt loop tests described above, surveillance of the impurity content of the salts during irradiation, and post-irradiation examination of the materials used as containers, can be conducted at the test bed. This surveillance will quantify corrosion rates and aid in determining suitable designs. New concepts can be conveniently tested before they become part of an operating spallation neutron source facility.

\section{E. Contamination of Fluid Systems with Transmutation Products}

Corrosion and spallation products enter coolant systems at spallation neutron source facilities. Radioactive contaminants must be effectively trapped in appropriate filters so the contaminants can be properly handled and disposed of. The goal is to mitigate the undesirable effects of these contaminants anywhere in the system. The test bed offers an opportunity to develop the equipment and procedures necessary to accomplish this task.

\section{F. Development of Surveillance Methods and Monitors of System Performance} Knowledge of the status of equipment in operation enables intelligent decisions to be made regarding the need for replacement or repair of a component. Such information is essential for any maintenance program in order to be able to provide reliable operations. In spallation neutron source environments, any monitor of system performance will be exposed to particle or electromagnetic radiation and thus suffer possible degradation. The proposed test bed offers an opportunity to systematically test surveillance equipment and develop methods that can be trusted. Areas of concern include temperature and flow measurements, coolant chemistry measurements, and behavior of materials irradiated 
during the operation of a facility. The removal and inspection of liquid metal target material can be used as a measure of the performance of the system, especially in terms of corrosion behavior. The test bed is essential for developing needed capabilities in the area of surveillance.

\section{G. In situ Determination of Heat Transfer Properties and Degradation with Time} Spallation neutron source target, moderator, reflector, and shield configurations are complex in terms of geometry as well as function. Before the target system is placed in a facility, the coolant flow characteristics need to be carefully measured. Because heat generation in spallation neutron source components is internal due to energetic particle interaction with electrons and atoms, simulated laboratory heat transfer measurements are difficult. A test bed with a prototypic radiation environment provides the necessary correct heat generation morphology and allows for the determination of the systems' heat transfer characteristics. As materials change dimension because of radiation exposure, and as corrosion products deposit on surfaces, heat transfer characteristics change. The test bed is effective in determining the magnitude of these changes, in concert with the measurements on coolant chemistry described above.

\section{H. Comparison of System Design with Performance-Refinement of Design Technique}

The test bed enables the comparison of designed performance with measured performance. Design refinements for future facilities can then be developed based on data obtained in carefully controlled test bed experiments.

\section{I. Development of Choppers and Other Equipment at Near-Target Locations} Short-pulse spallation sources (SPSS) use choppers to "frame" the pulses of neutrons. Some applications could benefit from having the choppers placed very near the neutron source, but the radiation environment near the target will degrade bearings and motor drivers. Use of the test bed will enable the development of components such as air bearings and air motors that can operate successfully in the high radiation environment.

\section{J. Development of Beam Diagnostics Equipment}

A continual need exists to improve the reliability of instrumentation used to determine the characteristics of the proton beam. This instrumentation includes beam profile monitors, current monitors, beam position monitors, and thermocouple systems for measuring 
component temperatures. A test bed will provide an excellent opportunity for comparing various techniques and developing and qualifying beam status instruments.

\section{MATERIALS PERFORMANCE ISSUES}

\section{A. Mechanical Properties Degradation}

The effects of atomic displacements in materials exposed to particle radiation are generally an increase in tensile strength, a decrease in ductility, and a decrease in toughness [6-9]. These effects are accelerated when impurities are introduced concurrent with the displacements. Spallation neutron source environments contain more energetic particles than fission reactor environments. This more energetic environment causes displacements and copious amounts of transmutation product impurities to be made concurrently. Data from fission reactor experience can be used only as a guide since impurity production in fission reactor environments is 10 to 100 times less than in spallation neutron source environments. A critical need exists to develop a database of information concerning materials radiation damage effects in spallation neutron source environments, in both a proton beam and an energetic spallation neutron flux. A test bed provides the proper environment for testing the long-term exposure of materials under controlled conditions. These tests could be performed simultaneously with the LPSS target system and the samples taken to high fluence when they remain in the facility over several operating periods.

\section{B. Physical Properties Degradation}

As transmutation products are deposited on material grain boundaries, thermal and electrical properties may be degraded. These degradation effects need to be quantified for candidate spallation neutron source materials irradiated in a prototypic environment.

One area requiring further development for spallation neutron source environments is the use of permanent magnets in areas where beam stability must be maintained in the event of electrical power anomalies (ensuring that a defocused beam does not focus to a spot size that would damage a beam entry window or target). The stability of the magnetic properties of materials must be tested in a prototypic environment.

\section{C. Dimensional Stability}

Concurrent atomic displacement damage and production of gases such as He lead to nucleation and growth of interstitial dislocation loops, voids, and gas bubbles in the 
material microstructure. After extended exposure, macroscopic dimensional changes occur. In some applications at spallation neutron source facilities, dimensional stability is essential. The magnitude of dimensional changes in candidate spallation neutron source materials needs to be determined and resistant materials need to be identified. A test bed that has a spallation neutron source prototypic environment is essential for conducting these studies.

\section{D. Thermal Stress from Thermal Strain at Low Frequency}

Nominal static stresses for spallation neutron source components can generally be held at acceptable levels. Of concern, however, is the effect of a rapid quench from operating temperature to coolant temperature caused by an accelerator shutdown. Operating accelerators experience such shutdowns several times a day, generally for a few seconds at each occurrence. Likely effects on mechanical behavior include cyclic stresses and crack propagation driven by those cyclic stresses as well as a synergistic effect from the concurrent production of point defects and transmutation product atoms. Another concern is the effect of the cyclic, thermally driven strain on the interface between cladding and target pins. These effects can be tested directly in the test bed on an ongoing basis, concurrent with other experiments.

\section{E. Shock-Related Stress from Short-Pulse, High-Intensity Operation}

The issue of stock-related stress has been raised for targets and beam entry windows operating in systems that use very intense, short pulses on the order of $20 \mathrm{~A}$ during a 250-ns pulse. The Manuel Lujan Jr. Neutron Scattering Center (MLNSC), which has a tungsten rod target, operates in this mode. The concern is that cracks may occur in a target rod as a result of the magnitude of a reflected tensile stress wave that produces an exceptionally high stress at the center of a rod. The test bed will use pulses that are 0.5 to $1.0 \mathrm{~ms}$ long, and is thus not appropriate for conducting shock-related stress tests. However, the tests can be initiated at the MLNSC and the test samples can later be irradiated to high dose at the test bed as a means of determining long-term effects after the postulated crack in the center of the rod has been formed.

\section{F. Synergetic Effects of Radiation and Environment}

Cooled metal surfaces of components in the proton beam at a spallation neutron source facility encounter an environment that is likely to be highly ionized. Mechanical property degradation processes, such as stress corrosion cracking, may be accelerated under these conditions. Studies with conventional coolants such as water, as well as studies with 
liquid metal and molten salts, are needed to quantify these effects in energetic charged particle beams and in a spallation neutron flux.

\section{G. Comparison of Spallation Neutron Source Performance Data to Fission Reactor Data}

Comparative studies of material property changes between fission reactor, spallation neutron, and proton irradiations would enhance the ability to determine whether the fission reactor materials database can be applied to spallation neutron source applications and, if so, to apply it. A test bed with a spallation neutron source environment where controlled experiments at high radiation dose can be performed is essential for these comparative studies.

\section{H. Basic Understanding of Atom Displacement Physics at High Recoil Energies}

Progress is being made on computer-based radiation damage and radiation effects calculations that predict the details of displacement cascades and microstructural evolution. The codes used for these calculations would benefit from accurate measurements of point defect production rates and other basic data in spallation neutron source environments. These measurements require a test bed with prototypic spallation neutron source radiation environments.

\section{I. Basic Understanding of Transmutation Product Morphology and the Effect on Properties}

Atoms formed from spallation transmutation reactions enter a metal alloy system as an impurity. The subsequent diffusion and reaction history, as well as the final fate of the impurities, are dependent on radiation rate, temperature, energy of atomic recoils, and other factors such as grain size. Development of alloys that can resist the detrimental effects of impurities requires first a basic understanding of the kinetics and chemistry of the governing processes during irradiation. Work in this area using a test bed with the proper radiation environment may lead to development of low activation, low neutron absorbing, thermally stable alloys for use at spallation neutron sources. 


\section{NEUTRONICS PERFORMANCE ISSUES}

\section{A. Production of Neutrons/Proton for Several Materials}

Neutron production in materials can be characterized at a power level below the 1-MW power level proposed for the test bed, but the overall neutron production efficiency of an "engineered" system will be measured during the systems tests described above. These measurements will require flight paths viewing appropriate parts of the target system.

\section{B. Production of Tritons/Proton for Several Materials}

Triton production can also be measured at lower power levels than are available at the proposed test bed. Again, when the measurements are made in conjunction with systems tests at the high power levels available at an LPSS test bed, excellent statistics would be possible. On-line testing of the separation of tritium from $\mathrm{He}$, as is needed in the APT program, would be possible by including appropriate gas-handling systems studies.

\section{C. Moderator Design Developments/Ultracold Neutron Source}

The development of cryogenic temperature moderators, particularly a moderator for the proposed ultracold neutron source, would benefit greatly from use of a test bed where the radiation environment produces the proper internal heat generation. The test bed can be configured to allow rapid modifications to the system as changes are warranted during testing. The test bed is essential to this work.

\section{D. Poison and Decoupling Studies}

The size of the irradiation volume at the test bed will allow testing of complete target system assemblies. Optimization studies can be accomplished and configuration changes can be made as the tests proceed. Configuration changes involve handling radioactive materials, and such changes would be possible because the LPSS facility will have extensive remote handling and hot cell equipment. Comparison of performance calculations to performance measurements can also be made.

\section{E. Blanket Design}

Blanket design studies are needed to optimize the design for APT and other ADTT facilities. As in the case of the decoupling studies, optimization can be accomplished and configuration changes can be made as the tests proceed. Comparison of performance calculations to performance measurements can also be made. 


\section{F. Transmutation Product Generation Measurements at High Fluence with}

Burnup and General Neutronics Code Verification and Benchmark Studies

Spallation neutron source target systems will be expected to perform to high fluences. At high fluences, high activity levels are a concern from the standpoint of waste disposal. At present, code predictions are used to determine the terminal activity and radioisotope inventory for irradiated components. At high dose, atom burnup is a factor. The computational codes should be validated at high fluence; validation can conveniently be accomplished at a test bed when radioisotope measurements are made following controlled high-fluence irradiations.

\section{TESTING METHODOLOGY}

The test bed facility will be arranged to provide maximum flexibility for performing the tests described above. Some of the features of the facility are shown in Figures 1 through 3. Remote handling procedures will be in place to modify test assemblies as the tests dictate or to replace and repair test assemblies. The flexibility and versatility of the facility and the prototypic radiation environment available at the proposed test bed are ideal for the development of spallation neutron source target stations.

\section{A. Short-term Performance Tests}

The following tests and experiments, described in detail above, would require use of the facility for a period of about one week:

- Measurement of neutron spectrum and flux using a flight path.

- Heat transfer measurements with in situ instrumentation.

- Full-scale systems performance evaluation and development.

- Basic corrosion parameter measurements.

- Performance evaluation of target system variables. These tests may require several operating periods with sufficient time between tests to effect suggested changes. 


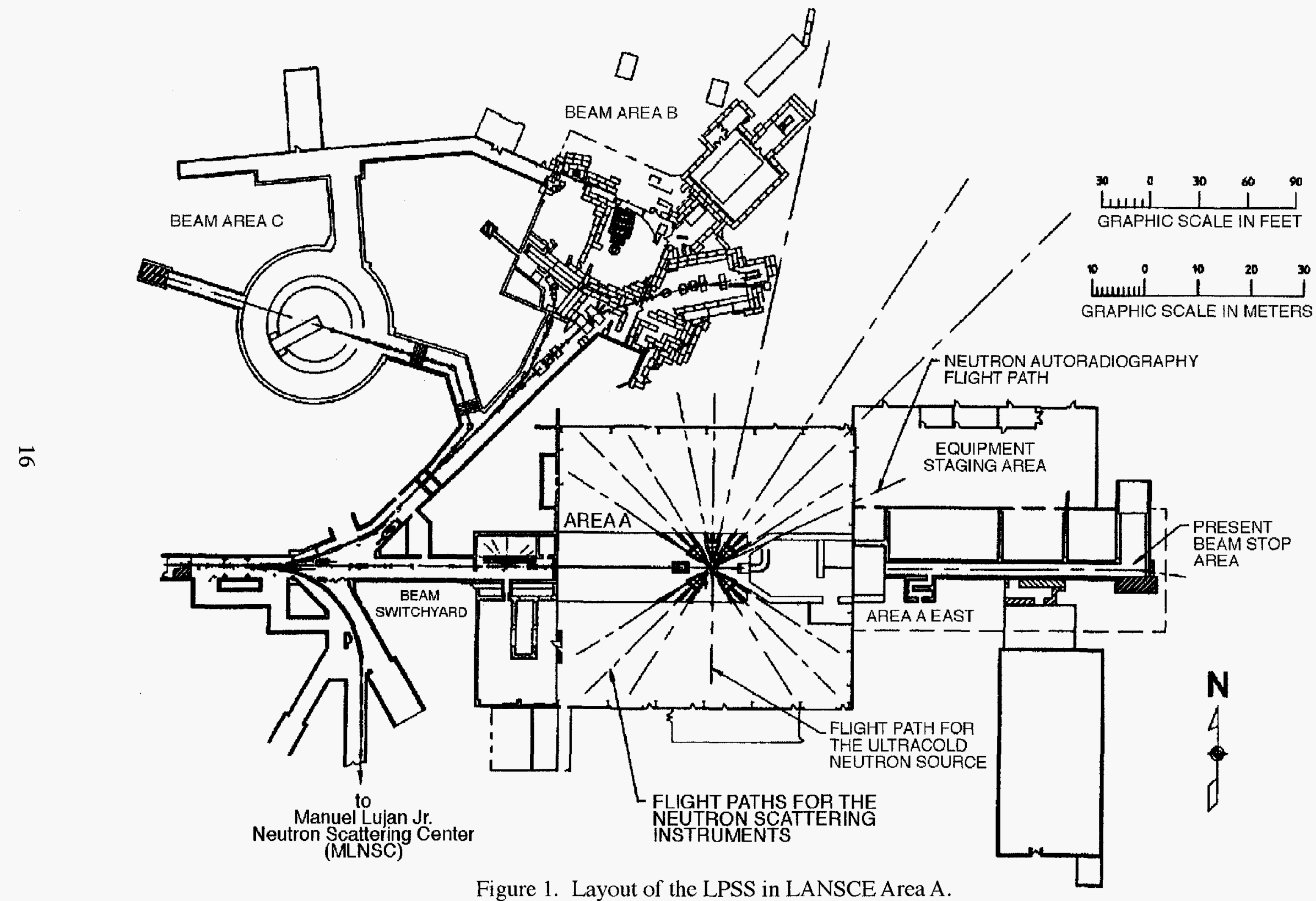




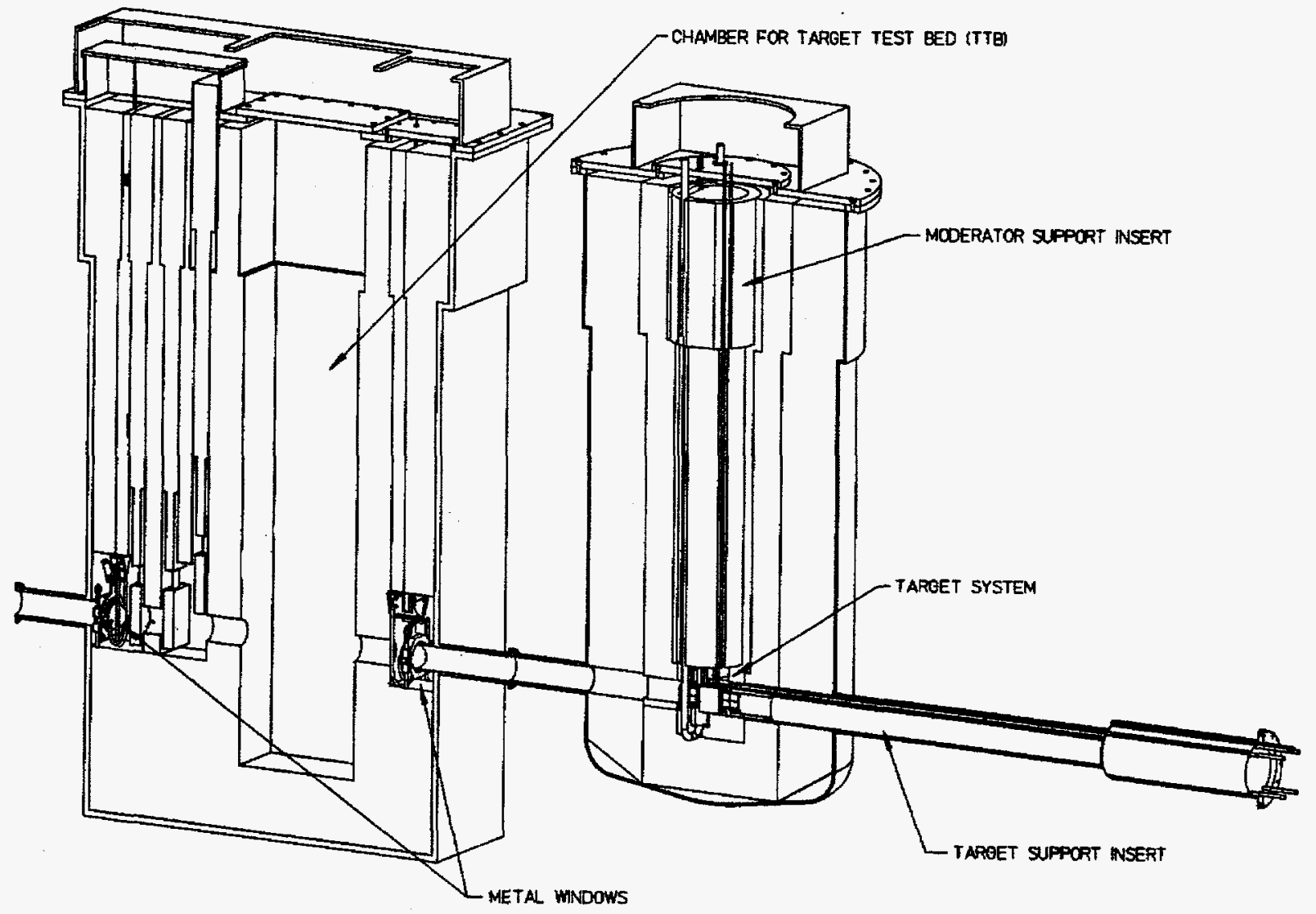

Figure 2. The target test bed in front of the LPSS target system.

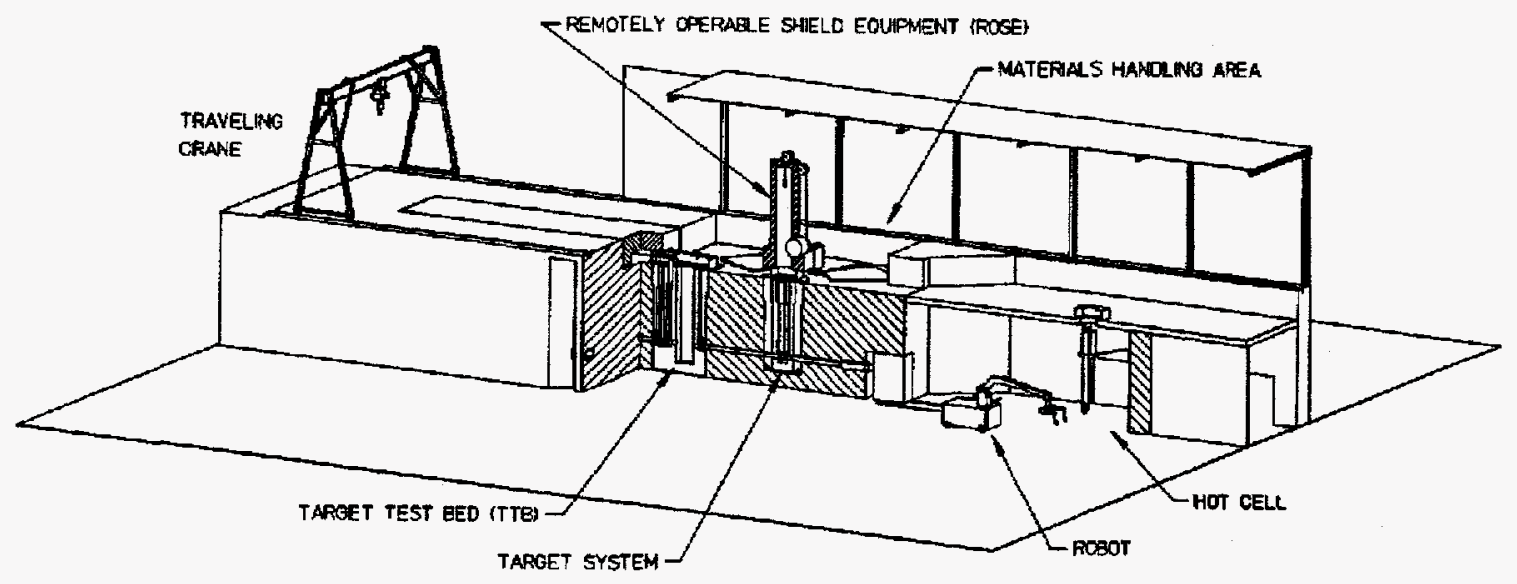

Figure 3. Remote handling equipment and hot cells. 


\section{B. Long-term Performance Tests}

Tests and experiments can be housed in the LPSS target area for extended periods of time. These tests and experiments include evaluation of

- corrosion at high fluence and long times;

- systems components lifetime (for pumps, filters, etc.);

- cleanup and filtration/isolation of spallation and corrosion products;

- long-term corrosion mitigation methods.

\section{C. Very Long-term Materials Exposure for Radiation Damage Effects Determination}

Determination of materials radiation damage effects will require long-term exposures. Materials will be placed in specially designed capsules that will be placed directly in the proton beam and in various locations in the neutron flux. The layout of these experiments will ensure that the experiments do not interfere with the LPSS target system and have no adverse effect on the LPSS neutronics performance.

It will be possible to examine materials after long-term exposures at working facilities, including the LPSS, because the test bed facility will be equipped with state-of-the-art materials testing and characterization facilities in the hot cell. The hot cell facilities could be used for testing materials that become available from spallation neutron sources worldwide.

\section{D. Use of Instruments at LPSS and MLNSC for Materials Characterization after Irradiation}

Use of neutron scattering and X-ray equipment available at the LPSS and MLNSC will greatly enhance the material characterization capability at the test bed. One chief advantage is that the facilities are all on a common site, so transport of radioactive materials on public roadways is not necessary. 


\section{SPALLATION NEUTRON SOURCES UNDER STUDY AND DEVELOPMENT}

There is presently a great deal of worldwide interest in spallation neutron sources, and several high-power pulsed neutron sources for research in neutron scattering and basic physics are in development or design. Very high power facilities (100 to $200 \mathrm{MW}$ ) are being considered for the transmutation of elements for the production of tritium, the mitigation of radioactive isotopes from fission reactor and defense-related waste, and the use of plutonium from weapons programs for a subcritical accelerator-driven energy production device. Each facility being studied has a unique design driven by performance requirements and the need for long-term reliable operation. The most prominent facilities are described below. The magnitude of the activity in the area of advanced spallation neutron sources serves as a measure of the impact a target testing and development facility would have in this field.

\section{A. Research Facilities}

6.A.1. European Spallation Source (ESS). The countries in the European community are collaborating on a 5-MW source that will be a general purpose neutron scattering and physics facility with a beam energy of $1.35 \mathrm{GeV}$. The system will be pulsed at $50 \mathrm{~Hz}$. The designers of this facility are concerned about the integrity of beam entrance windows and stationary solid targets. The development needs at this facility include the determination of neutronics performance and system operating parameters for rotating solid targets and liquid metal target systems. In addition, the performance of beam entry windows needs to be assessed. This project is in the conceptual design stage.

\section{A.2 Short-Pulse Spallation Source (SPSS). There are at least three competing concepts for an SPSS with a proton beam energy in the 0.8 to $1.6 \mathrm{GeV}$ range. Pulse lengths of 100 to $300 \mathrm{~ns}$ are being considered. The various designs consider two target stations, with one operating at a power level of $1 \mathrm{MW}$ and the other at a power level up to $5 \mathrm{MW}$. Pulse rates of 20,40 , and $60 \mathrm{~Hz}$ are being considered. Advanced concepts for moderators are also being studied.}

Development of an SPSS would benefit greatly from the opportunity to test target system configurations and the effect of geometry on neutronic performance. Testing of engineered systems is also very important for the high-power systems needed at an SPSS 
facility. The requirement for long-life components and reliable operation leads to the need for high-fluence materials radiation damage effects studies. The studies would be conducted in parallel with the neutronics and engineering systems tests.

6.A.3 Long-Pulse Spallation Source (LPSS). It is being proposed that the LPSS facility use the LANSCE accelerator and that the LPSS target system be placed in the large experimental hall at LANSCE after the nuclear physics program is completed in FY 96. The power at the facility will be 0.8 to $1.0 \mathrm{MW}$ and could perhaps be taken to 5 to $10 \mathrm{MW}$. The current beam energy is $800 \mathrm{MeV}$ and the system is pulsed at 60 to $120 \mathrm{~Hz}$ with a 0.5 - to 1.0 -ms pulse length. As many as 15 flight paths in Area A at LANSCE (see Figure 1) will service a suite of neutron scattering instruments. Provisions for neutron radiography, studies of radiation damage to materials, ultracold neutron production, and provisions for other applications will be made. Advanced target system handling procedures are being studied for use at this facility. The test bed for spallation neutron source target system studies can be included at the LPSS, as shown in Figures 2 and 3.

\section{A.4 Target System Development at the Manuel Lujan Jr. Neutron Scattering} Center (MLNSC). This facility is being upgraded and extended in power from $80 \mathrm{~kW}$ to $160 \mathrm{~kW}$. The facility operates at $800 \mathrm{MeV}$ at 20 to $40 \mathrm{~Hz}$ with a 250-ns long pulse.

Advanced moderator development is desired to enhance instrument capability. Provision is being made for studies of low-dose radiation damage to materials to determine basic radiation damage parameters. Design of services and remote handling are aimed at ensuring a reliable 9 months of operation each year.

A target system upgrade at MLNSC features the development of a target design based on encapsulated pure tungsten rods 0.5 to $1.0 \mathrm{~cm}$ in diameter. Further development of the facility will benefit from use of the test bed described in this document.

\section{A.5. Target System Development at the Swiss Spallation Neutron Source (SINQ).} SINQ will be a 0.9-MW, 590-MeV, 1.5-mA beam current, continuous wave machine. Target development studies are presently underway, but without radiation exposures. Plans call for using $\mathrm{Zr}$ rods when the facility opens, changing to $\mathrm{Pb}$ rods when operating experience allows, and finally changing the target to liquid $\mathrm{Pb}$. There are plans to use some of the volume in the target chamber as a long-term materials testing facility. In addition, the developers of the facility intend to incorporate advanced remote handling 
techniques and study methods to mitigate the effects of contaminated coolant water circuits. There is also a strong desire at SINQ to develop liquid metal targets; the test bed proposed here would support these studies.

In a collaboration between SINQ and LANSCE/MLNSC, beam entry windows made of stainless steel alloy HT-9 were irradiated at LANSCE to a dose exceeding $10^{21}$ protons $/ \mathrm{cm}^{2}$. Plans for measuring the radiation effects on the mechanical properties of these materials are now being made.

\section{A.6. Target System Development at ISIS at Rutherford-Appleton Laboratory. ISIS is an $800-\mathrm{MeV}, 200-\mu \mathrm{A}$ machine used for neutron scattering and basic physics research at Rutherford-Appleton Laboratory in the United Kingdom. Their facility staff has experience with $U$ and $T a$ targets and has developed advanced remote handling techniques for replacing these highly activated components. Further development of the clad targets for this facility would benefit greatly from the test bed being proposed.}

\section{A.7. Intense Proton-Neutron Source (IPNS) Upgrade. Argonne National Laboratory} is proposing to upgrade its IPNS; specifically, it is proposed that this SPSS utilize an energy of 0.6 to $3.6 \mathrm{GeV}$ with $0.7 \mathrm{~mA}$ of current incident on each of two targets. The upgrade studies may produce a 5-MW source design, but emphasis will initially be placed on developing a 1-MW source. Remote handling techniques are planned for the facility to allow rapid repair and replacement of failed target components. Target development and the development of remote handling techniques could benefit from studies at the LPSS test bed.

\section{A.8. Conceptual Design for the Austrian Spallation Source (AUSTRON).}

AUSTRON proposes a pulsed source using a $1.6-\mathrm{GeV}, 25-\mathrm{Hz}$ accelerator beam with 60 -ns pulses. Total power is expected to be greater than $1 \mathrm{MW}$. The candidate target material now being considered is a tungsten alloy (W-5\% Re). In the proposed design, the target is cooled on the edges so that the coolant water does not directly interact with the proton beam. The AUSTRON target design could also benefit from tests in the LPSS test bed facility.

\section{A.9 Engineering Test Accelerator (ETA). There is an initiative at the National Laboratory for High-Energy Physics (KEK) in Japan to build a test bed for future spallation neutron source developments. This facility would operate at $1.5 \mathrm{GeV}$ and}


$10 \mathrm{~mA}$ and would be equipped to perform high-power neutronic studies. The ETA facility would be used for systems studies and materials performance measurements much like those described in this document.

\section{B. Large-Scale Production Facilities}

6.B.1. Accelerator Production of Tritium (APT). APT is to be a continuous wave accelerator at $1 \mathrm{GeV}$ and $200 \mathrm{~mA}$ whose purpose is to produce tritium from neutron capture on ${ }^{3} \mathrm{He}$. The design features a target made of tungsten rods and a blanket assembly containing heavy water as a moderator. Reliability and availability are important concerns for this facility. One test considered especially important is the evaluation of the corrosion of metal surfaces that are in contact with water coolant while being irradiated with protons. The expected lifetime of components is an issue and plans are being made for a surveillance program that will monitor materials performance on an ongoing basis. Some materials tests are planned at the LANSCE beam stop irradiation facility in 1995-1997. Neutron production and tritium production rates will be measured to quantify the efficiency of the system and to serve as benchmarks for the Los Alamos Code System used to predict performance. The ongoing development of the APT program would benefit from a spallation neutron source test bed.

\section{B.2 Accelerator-Driven Transmutation Technologies (ADTT). Future ADTT} accelerators may be continuous wave at an energy of 0.8 to $1.6 \mathrm{GeV}$ and a current of 10 to $20 \mathrm{~mA}$. Development needs include liquid metal and molten salt loop tests that consider corrosion and mass transport. The effects of charged particle radiation on liquid metal and molten salt performance also need to be studied. Materials lifetime and compatibility with liquid metal and molten salt need to be established. An understanding of the materials performance will allow proper assessment of the safety concerns in dealing with these materials. Again, the usefulness of a spallation neutron source test bed to this project is evident.

\section{C. Workshop on Materials for Spallation Neutron Sources, Los Alamos,} February 1995.

Over 100 persons attended an international meeting on materials for spallation neutron sources that was held at Los Alamos in early February 1995. There was general agreement that materials lifetime in spallation neutron source environments was a major concern and that prototypic data were lacking. It was determined that a dedicated 
spallation neutron source materials irradiation facility was a necessary ingredient toward achieving the needed materials data.

\section{REFERENCES}

1. A. Carne, T. A. Broome, J. R. Hogston, and M. Holding, "The ISIS Target," in Proceedings of the 10th Meeting of the International Collaboration on Advanced Neutron Sources (ICANS X), Institute of Physics Conference Series Number 97 (1989), p. 79.

2. T. A. Broome and M. Holding, "Remote Handling for an ISIS Target Change," Proceedings of the 10th Meeting of the International Collaboration on Advanced Neutron Sources (ICANS X), Institute of Physics Conference Series Number 97 (1989), p. 585.

3. "APT He-3 Target/Blanket Topical Report," Los Alamos National Laboratory report LA-12670-MS, Volume I, Revision 1 (March 1994).

4. R. D. Werbeck, Los Alamos National Laboratory, private communication to W. F. Sommer on experience with the LAMPF high-power target stations.

5. P. Cloth, D. Filges, R. D. Neef, "Calculation of the Time Dependent Dose Rate of the SNQ-Target Cooling Water and the Moderator Water for Radiolysis Considerations," Kernforschungsanlage Juelich Report, SNQ 3 J1 BH 10128 (December 1994).

6. John F. Kircher and Richard E. Bowman, Effects of Radiation on Materials and Components (Reinhold Publishing Corporation, New York, 1964).

7. M. S. Wechsler, J. F. Stubbins, W. F. Sommer, P. D. Ferguson, and E. H. Farnum, "Selection and Qualification of Materials for the Accelerator Transmutation of Waste Project," Los Alamos National Laboratory report LA-UR-92-1211 (June 1992).

8. John R. Weeks, Carl J. Czajkowski, and Paul Tichler, "Effects of High Thermal and High Fast Fluences on the Mechanical Properties of Type 6061 Aluminum at HFBR," ASTM STP 1046 (1990), pp. 441-452. 
9. K. Farrell and R. T. King, "Tensile Properties of Neutron-Irradiated 6061 Aluminum Alloy in Annealed and Precipitation-Hardened Conditions," ASTM STP 683 (1979), pp. 440-449.

\section{ACRONYM LIST}

ADTT Accelerator-Driven Transmutation Technologies

APT Accelerator Production of Tritium

$\begin{aligned} \text { AUSTRON } & \text { Austrian Spallation Source } \\ \text { ESS } & \text { European Spallation Source } \\ \text { ETA } & \text { Engineering Test Accelerator } \\ \text { IPNS } & \text { Intense Proton-Neutron Source (Argonne National Laboratory) } \\ \text { ISIS } & \text { Rutherford-Appleton Laboratory (United Kingdom) } \\ \text { KEK } & \text { National Laboratory for High-Energy Physics (Japan) } \\ \text { LANSCE } & \text { Los Alamos Neutron Science Center (formerly known as LAMPF) } \\ \text { LPSS } & \text { Long-Pulse Spallation Source } \\ \text { MLNSC } & \text { Manuel Lujan Jr. Neutron Scattering Center } \\ \text { ROSE } & \text { Remotely Operable Shield Equipment } \\ \text { SINQ } & \text { Schweizerisches Institute für Nuklearforschung Quelle } \\ & \text { (Swiss Institute of Nuclear Research Source) } \\ \text { SPSS } & \text { Short-Pulse Spallation Source } \\ \text { TTB } & \text { Target test bed }\end{aligned}$

